\title{
HALF FACTORIAL DOMAINS ${ }^{1}$
}

\author{
BY ABRAHAM ZAKS
}

Communicated by Barbara Osofsky, May 3, 1976

1. Introduction. Let $R$ be communicative domain with 1 . We call $R$ a Half-Factorial Domain (HFD) provided the equality $\Pi_{i=1}^{n} x_{i}=\prod_{j=1}^{m} y_{j}$ implies $m=n$, whenever the $x$ 's and the $y$ 's are nonunit and irreducible elements of $R$. The study of HFD's is motivated in part by a result in number theory of Carlitz [1], and was raised by Narkiewicz [4]. For a Krull domain $R$, we denote by $C(R)$ the ideal class group of $R$. We identify the ideal $P$ with its image in $C(R)$. The ring $\mathbf{Z}$ denotes the ring of integers.

A Dedekind domain $R$ is called special, if whenever a prime $P$ equals (in $C(R)$ ) a product of primes $Q_{1} \cdots Q_{t}$, it already equals one of the $Q$ 's (in $C(R)$ ).

2. General criteria. Remark that if $R$ is an HFD, then for every $r \neq 0$ if $r$ is a nonunit in $R, w(r)=$ number of irreducible factors in a complete decomposition for $r\}$ is a well-defined function on $\hat{R}=\{r \mid \neq 0, r$ nonunit, $r \in R\}$ into the positive integers, such that $w(r s)=w(r)+w(s)$ for $r, s \in \hat{R}$, and $w(r)=1$ iff $r$ is an irreducible element in $R$. Conversely, the existence of such a function implies that $R$ is an HFD.

Proposition 1. Let $R$ be a Dedekind domain with torsion class group. Then $R$ is an HFD iff whenever $P_{1}, \ldots, P_{t}$ are prime ideals so that $P_{1}^{n_{1}} \cdots P_{t}^{n_{t}}=R x$, there exists a subproduct $P_{1}^{m_{1}} \cdots P_{t}^{m_{t}}=R y, m_{i} \leqslant n_{i}$, where $y$ is an irreducible element such that $\left(m_{1} / s_{1}\right)+\cdots+\left(m_{t} / s_{t}\right)=1$, where $s_{i}$ is the order of $P_{i}\left(s_{i}=1\right.$ if $\left.P_{i}=R z\right)$.

Proposition 2. Suppose that in the Dedekind domain $R$, for every prime ideal $M$ there exists a prime ideal $N$ so that $M N$ is a principal ideal. Then $R$ is an HFD iff $C(R)$ is 2-elementary and $R$ is a special Dedekind domain, or else $C(R)$ is isomorphic to $Z$, and a prime ideal $P$ exists so that for every prime ideal $M$ exactly one of the following equalities holds: (i) $M \equiv P$, (ii) $M P \equiv R$, (iii) $M \equiv R$.

The following theorem leads to a class of Dedekind domains that are HFD $13 \mathrm{D} 15$.

AMS (MOS) subject classifications (1970). Primary 10A25, 13F99; Secondary 12A45,

Key words and phrases. Dedekind domains, Krull domains, ideal class-group.

${ }^{1}$ This research was supported in part by the Israel Academy of Sciences and Humanities-The Israel commission for basic research, grant M-C-8.

Copyright $\odot$ 1976, American Maticmatical Society 
with a given finitely generated abelian group as its ideal class group. The existence of domains in this class is a consequence of the Claborn construction of Dedekind domains with a prescribed ideal class group (e.g. [2], [3]).

Theorem 3. Let $R$ be a Dedekind domain with $C(R)$ a finitely generated abelian group. Let $C_{1} \oplus \cdots \oplus C_{n}$ be a decomposition of $C(R)$ into cyclic groups. If (a) prime ideals $P_{1}, \ldots, P_{n}$ exist so that $P_{i}$ generates $C_{i}$, (b) for every prime $Q$ in $R$, if $Q$ is a nonprincipal prime ideal, then for some $i$ either $Q \equiv P_{i}$ or else $Q P_{i} \equiv R$, and (c) if for some $i, Q P_{i} \equiv R$, then $C_{i}$ is isomorphic to $Z$. Then $R$ is an HFD.

3. The cyclic case. We start with a question:

Definition. A set of positive integers $\left\{p_{1}, \ldots, p_{t}\right\}$ is a splittable set if whenever $n_{1}, \ldots, n_{t}$ are positive integers so that $\left(n_{1} / p_{1}\right)+\cdots+\left(n_{t} / p_{t}\right)$ is an integer, there exist positive integers $m_{1}, \ldots, m_{t}$ so that $m_{i} \leqslant n_{i}$ and $\left(m_{1} / p_{1}\right)$ $+\cdots+\left(m_{t} / p_{t}\right)=1$

Question. Is every set of positive integers splittable?

As for the general cyclic case we have

THEOREM 4. If $C(R)$ is cyclic of order $n$, and if there exists a prime $P$ that generates $C(R)$, then $R$ is an HFD iff (a) for every prime $Q$ that is nonprincipal there exists an integer $m$ so that $m$ divides $n$, and $Q \equiv P^{m}$, and (b) if $\left(m_{1}, \ldots, m_{t}\right)$ is a complete set of integers so that for every prime $P$ in $R$ there exists an $i, 1 \leqslant i \leqslant t$, so that $m_{i}$ is the order of $P$ in $C(R)$, then this set is a splittable set.

Passing to the case $C(R)$ is cyclic of prime order $p$ we obtain

Corollary 5. Let $R$ be a Dedekind domain whose class group is a cyclic p-group. Then $R$ is an HFD iff for every pair of prime ideals $M$ and $Q$, there exists an integer $n$ so that $M \equiv Q^{p^{n}}$ or else $Q \equiv M^{p^{n}}$.

COROLlary 6. Let $R$ be a Dedekind domain whose class group is a cyclic p-group. If $R$ is an HFD then every overring of $R$ is an HFD.

REMARK a. The converse to Corollary 6 is false in general.

REMARK b. There exist Dedekind domains that are HFD, for which $C(R)$ is cyclic of order $n$, but no prime ideal generates $C(R)$.

REMARK C. Similar results hold in case $C(R)=z_{p^{\infty}}$, thus leading to Dedekind HFD's for which $C(R)=C_{1} \oplus \cdots \oplus C_{n}$, where each $C_{i}$ is cyclic or an indecomposable torsion divisible group.

REMARK d. If $C(R)$ is a cyclic group of order $n$ and if $R$ is an HFD, then it is false, in general, that every overring of $R$ is an HFD. However, if $n$ is a prime, and $R$ has no prime elements then every overring of $R$ is a PID. The converse does not necessarily hold. 
THEOREM 7. Let $R$ be a Dedekind domain with $C(R)$ a 2-elementary group. Then $R$ is an HFD iff $R$ is a special ring.

We close with an interesting class of Krull domains that are HFD.

THEOREM 8. Let $R$ be a Krull domain. If $C(R)=Z / 2 Z$, then $R$ is an HFD.

THEOREM 9. Let $R$ be a Krull domain. The ring of polynomials over $R$ is an HFD iff either $R$ is a UFD or else $C(R)=Z / 2 Z$.

Corollary 10. A Krull domain $R$ with $C(R)=Z / 2 Z$ is the intersection of two Krull domains $S, T, R=S \cap T$, where $S$ is a UFD, $T$ is an HFD all of whose proper flat overrings are UFD, and both $S$ and $T$ are localizations of $R$ within its field of quotients.

REMARK 1. Most of the results as stated for Dedekind HFD can be proved for Krull HFD with slight modifications.

REMARK 2. We do not know if Krull HFD exist, except those mentioned.

Remark 3. The domain $Z[\sqrt{-3}]$ is an HFD which is not a Krull domain.

Proofs and more details will appear elsewhere.

\section{REFERENCES}

1. L. Carlitz, A characterization of algebraic number fields with class number two, Proc. Amer. Math. Soc. 11 (1960), 391-392. MR 22 \#2603.

2. L. Claborn, Specified relations in the ideal group, Michigan Math. J. 15 (1968), 249-255. MR $37 \# 4057$.

3. R. H. Fossum, The divisor class group of a Krull domain, Springer-Verlag, Berlin and New York, 1973.

4. W. Narkiewicz, Some unsolved problems, Bull. Soc. Math. France 25 (1971), 159164. ISRAEL

DEPARTMENT OF MATHEMATICS, ISRAEL INSTITUTE OF TECHNION, HAIFA, 